\title{
Constructing Nucleon Operators on a Lattice for Form Factors with High Momentum Transfer
}

\author{
Sergey Syritsyn ${ }^{* a, b, c}$, Bernhard Musch $^{d}$, Ajrun Gambhir $^{a, e}$, Kostas Orginos $^{a, e}$, \\ ${ }^{a}$ Thomas Jefferson National Accelerator Facility, 12000 Jefferson Avenue, Newport News, VA \\ 23606, USA \\ ${ }^{b}$ RIKEN/BNL Research Center, Brookhaven National Laboratory, Upton, NY 11973, USA \\ ${ }^{c}$ Department of Physics and Astronomy, Stony Brook University, Stony Brook, NY 11794, USA \\ ${ }^{d}$ Institut fr̈ Theoretische Physik, Universität Regensburg, 93040 Regensburg, Germany \\ ${ }^{e}$ Department of Physics, College of William and Mary, Williamsburg, VA 23187-8795, USA \\ E-mail: ssyritsyn@quark.phy.bnl.gov
}

\begin{abstract}
We present preliminary results of computing nucleon form factor at high momentum transfer using the "boosted" or "momentum" smearing. We use gauge configurations generated with $N_{f}=2+1$ dynamical Wilson-clover fermions and study the connected as well as disconnected contributions to the nucleon form factors. Our initial results indicate that boosted smearing helps to improve the signal for nucleon correlators at high momentum. However, we also find evidence for large excited state contributions, which will likely require variational analysis to isolate the boosted nucleon ground state.
\end{abstract}

34th annual International Symposium on Lattice Field Theory

24-30 July 2016

University of Southampton, UK

\footnotetext{
*Speaker.
} 


\section{Introduction}

Vector form factors are among the most important features of the nucleon describing its distributions of charge and magnetization densities,

$$
\left\langle P+q\left|\bar{q} \gamma^{\mu} q\right| P\right\rangle=\bar{u}_{P+q}\left[F_{1}\left(Q^{2}\right) \gamma^{\mu}+F_{2}\left(Q^{2}\right) \frac{i \sigma^{\mu v}}{2 M_{N}}\right] u_{P},
$$

where $F_{1,2}$ are Dirac and Pauli form factors and $Q^{2}=-q^{2}>0$ is the momentum transfer squared in elastic nucleon-photon scattering. Although the proton and neutron form factors have been studied extensively, many puzzles still remain. The form factors behavior at high momenta is expected to transition to perturbative QCD scaling [1,2], which has not been observed yet. The ration the proton electric and magnetic form factors may cross zero around $Q^{2}=7 \ldots 8 \mathrm{GeV}^{2}$, putting constraints on nucleon models in the Dyson-Schwinger framework. There is a large qualitative difference of contributions of $u$ - and $d$-quarks to both the $F_{1}$ and $F_{2}$ form factors[3]; understanding this phenomenon may also shed light on the internal organization of the nucleons. Upcoming experimental program at JLab will measure the nucleon form factors up to $Q^{2} \approx 18 \mathrm{GeV}^{2}$.

Calculation of high-momentum form factors on a lattice is complicated for a number of reasons. First, the signal-to-noise ratio is worse [4] for states at high momentum and hence energy. Second, the energy gaps between ground and excited states become smaller with increasing momentum, and higher statistical precision is necessary to perform excited-state analysis. Thus, reduction of the stochastic noise in nucleon structure calculations is crucial for computing highmomentum form factors. Such techniques are also important for computing TMDs and PDFs on a lattice, which require high-momentum nucleon initial and final states as well. Calculation of highmomentum form factors is a first step in studying high-momentum nucleon states on a lattice and validating the necessary methodology.

\section{Smearing for large momentum form factors}

The traditional Gaussian (Wuppertal) smearing is a method to create smooth covariant distributions for quark sources. Covariance is important because gauge-noninvariant contributions to hadron correlators increase statistical uncertainty. The width of quark sources is typically tuned to increase the overlap with the ground state of the studied hadron and suppress contributions of its excited states. The simple Gaussian shape is, however, not optimal when one has to construct a hadron interpolating field with large momentum, which is evident from a free-field example

$$
\mathscr{S}_{\text {at-rest }}=\exp \left[-\frac{w^{2}}{4}(i \vec{\nabla})^{2}\right] \sim \exp \left[-\frac{w^{2} \vec{k}_{\text {lat }}^{2}}{4}\right]
$$

demonstrating that the amplitude of a boosted quark in a Gaussian source falls off with momentum. For a nucleon field at large momentum, each of the component quarks may carry substantial momentum, and the suppression (2.1) may be an obstacle to performing calculations for highmomentum form factors. Indeed, the statistical precision of form factors on a lattice is typically much worse for higher momenta. It has been proposed $[5,6]$ that the boosted, or "momentum" 
smearing can improve the overlap with large-momentum states. The basic idea is to replace the smearing kernel (2.1) with a momentum-shifted one,

$$
\mathscr{S}_{\vec{k}_{0}}=\exp \left[-\frac{w^{2}}{4}\left(-i \vec{\nabla}-\vec{k}_{0}\right)^{2}\right] \sim \exp \left[-\frac{w^{2}\left(\vec{k}_{\text {lat }}-\vec{k}_{0}\right)^{2}}{4}\right]
$$

so that the smoothed quark wave function retains the average momentum $\vec{k}_{0}$ (see Fig. 1(left)):

$$
\left[\mathscr{S}_{\vec{k}_{0}}(\psi)\right]_{x}=e^{+\vec{k}_{0} \vec{x}} \mathscr{S}\left(e^{-\vec{k}_{0} \vec{y}} \psi_{y}\right) \sim e^{+\vec{k}_{0} \vec{x}} \cdot \text { smooth fcn. }(x) .
$$

Implementation of the boosted Gaussian smearing kernel (1) is similar to treating twisted boundary conditions (thus one can also boost by "fractional" momenta on a lattice):

$$
\left[\mathscr{S}_{\vec{k}_{0}}\right]_{x, y}=e^{+i \vec{k} \vec{k}_{0} \vec{x}}[\mathscr{S}]_{x, y} e^{-i \vec{k}_{0} \vec{y}} \quad \Leftrightarrow \quad \Delta_{x, y} \rightarrow e^{+i \vec{k}_{0} \vec{x}} \Delta_{x, y} e^{-i \vec{k}_{0} \vec{y}} \quad \Leftrightarrow \quad U_{x, \mu} \rightarrow e^{-i \vec{k}_{\mu}} U_{x, \mu}
$$

The momentum $\vec{k}_{0}$ is a tunable parameter for quark smearing. Naively, one could expect that one should select $\vec{k}=\frac{1}{3} \vec{p}_{N}$ for a baryon. However, it has been shown that larger momenta $\vec{k}_{0}$ improve the signal [6]. In this initial study we have only studied the "naive" $\vec{k}_{0}=\frac{1}{3} \vec{p}_{N}$. To achieve the highest momentum transfer $Q^{2}$ on a lattice at minimal nucleon energies, we arrange nucleon momenta and the smearing "boost" back-to-back (the Breit frame, Fig. 1(right)).
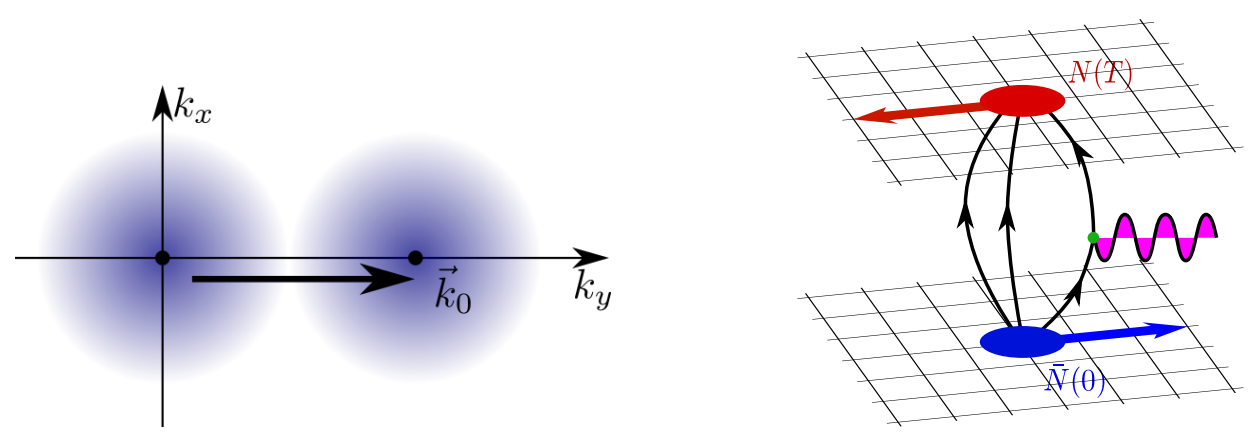

Figure 1: (Left) Boosted smearing shifts the "origin" of the approximately Gaussian-shaped quark source distribution. (Right) Breit frame for maximizing the momentum transfer.

\section{Results}

We perform our initial studies on two isotropic gauge ensembles with $N_{f}=2+1$ dynamical Wilson-clover fermions generated by the JLab group (Tab. 1). On each ensemble, we compute nucleon-current correlation functions with boosted quark sources and with five source-sink separations $t_{\mathrm{sep}}=(8 \ldots 12) a=(0.75 \ldots 1.13) \mathrm{fm}$ on the fine ensemble and $t_{\mathrm{sep}}=(6 \ldots 10) a=$ $(0.76 \ldots 1.27) \mathrm{fm}$ on the coarse ensemble. On both ensembles, the two largest separations do not have sufficient statistical precision and are omitted from the current analysis. The quark smearing is done with the "boost" momentum $\vec{k}=( \pm 1,0,0)$ on the fine ensemble and with $k_{0}=( \pm 1, \pm 1,0)$ on the coarse ensemble, to obtain approximately the same momentum in physical units, and the sink momentum is set to $\vec{p}_{N 0}=3 \vec{k}_{0}$ in both cases. In the Breit frame, the momentum transfer is thus $Q^{2}=4 \vec{p}_{N 0}^{2} \approx 6.1 \mathrm{GeV}^{2}$ on the fine ensemble and $Q^{2}=4 \vec{p}_{N 0}^{2} \approx 6.7 \mathrm{GeV}^{2}$ on the coarse ensemble. 
Table 1: Dynamical Wilson-clover ensembles used in this work. The pion and nucleon masses and lattice spacings are taken from Refs. [7, 8].

\begin{tabular}{l|ccc|ccc}
\hline \hline$L_{x}^{3} \times L_{t}$ & $a[\mathrm{fm}]$ & $m_{\pi}[\mathrm{MeV}]$ & $a M_{N}$ & conf & samp/conf & stat \\
\hline $32^{3} \times 64$ & $0.094(1)$ & $285(3)$ & $0.464(4)$ & 240 & 64 & 15,360 \\
$32^{3} \times 96$ & $0.127(2)$ & $278(3)$ & $0.619(3)$ & 210 & 96 & 20,160 \\
\hline \hline
\end{tabular}

In Figure 2 we compare the effective energies computed with ordinary (filled symbols) and boosted (open symbols) quark source smearing with two values of smearing "width" $w=4.96$ and $w=6.56$. In both cases, the boosted smearing yields substantially more precise results for the effective energy compared to the regular smearing at the largest momentum $\vec{p}_{N}=3 \vec{k}_{0}$. Therefore, calculations of nucleon structure with regular smearing at this momentum would be dominated by noise already at $t_{\text {sep }}=8 \mathrm{a}$. The signal for the effective energy becomes even worse when the width of the regular smearing is increased (Fig. 2(right)) in agreement with Eq. (2.1), whereas the boosted smearing data show stable values and smaller errors, indicating that the values of the smearing width "bracket" the width optimal for the overlap with the ground state. For the calculation of form factors, we choose the smearing width $w=5.55$.
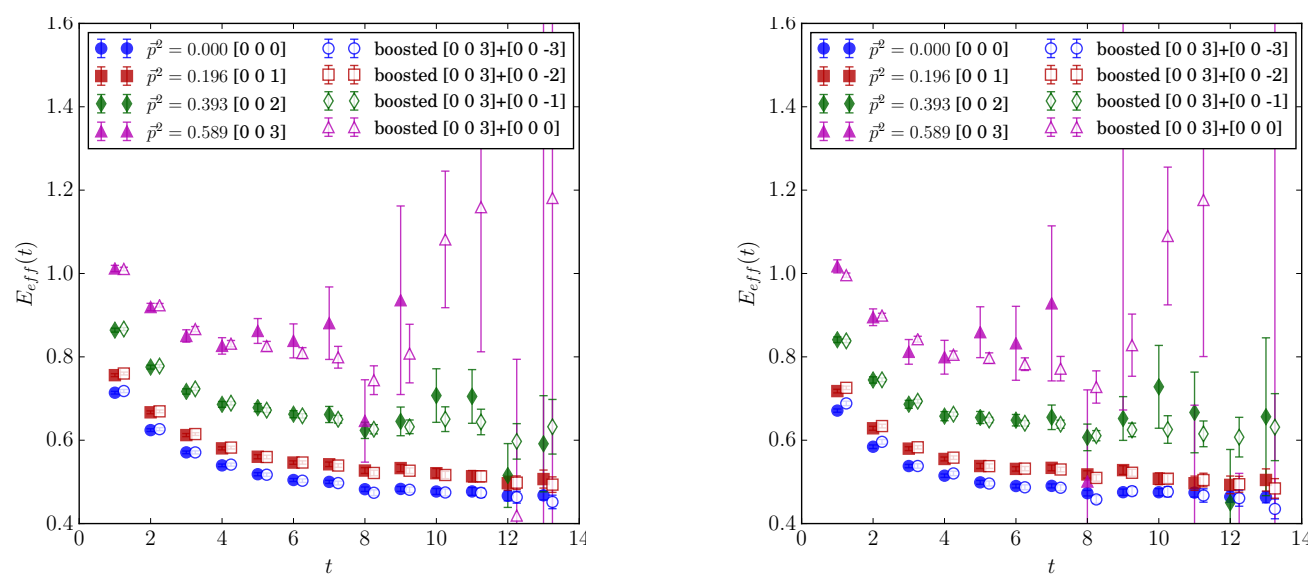

Figure 2: Effective energy plots from boosted and ordinary smearing with different width(number of smearing steps): $w=4.96(N=40)$ (left) vs $w=6.56(N=70)$ (right) on the $a=0.094 \mathrm{fm}$ ensemble.

In Figure 3 we show the two-state fits to the two-point functions with momenta up to $3 \vec{k}_{0}$ for both ensembles. The effective energy plateaus are typically reached by $t=7 a$ for the fine and $t=6 a$ for the coarse ensemble. We also show the data from correlation functions with smearing boosted in the direction opposite to the momentum on the right for comparison.

First we discuss quark-connected contributions to the form factors. Due to limited space, we concentrate on the fine $a=0.094 \mathrm{fm}$ ensemble. Elastic nucleon form factors at large momentum transfer $Q^{2} \gtrsim 1 \mathrm{GeV}^{2}$ decrease with $Q^{2}$ rapidly as predicted by the perturbative scaling [1]. In Figure 4 we show contributions of the $u$ and $d$ quarks to the form factor $F_{1}$ multiplied by $Q^{4}$.We note that although the dependence on the momentum $Q^{2}$ is in qualitative agreement with the flavorseparated form factors [3], the lattice data overshoots experiment by a factor of (3 ..4), which may be ascribed to excited state effects. Indeed, the data in Fig. 4 show substantial variation with the 

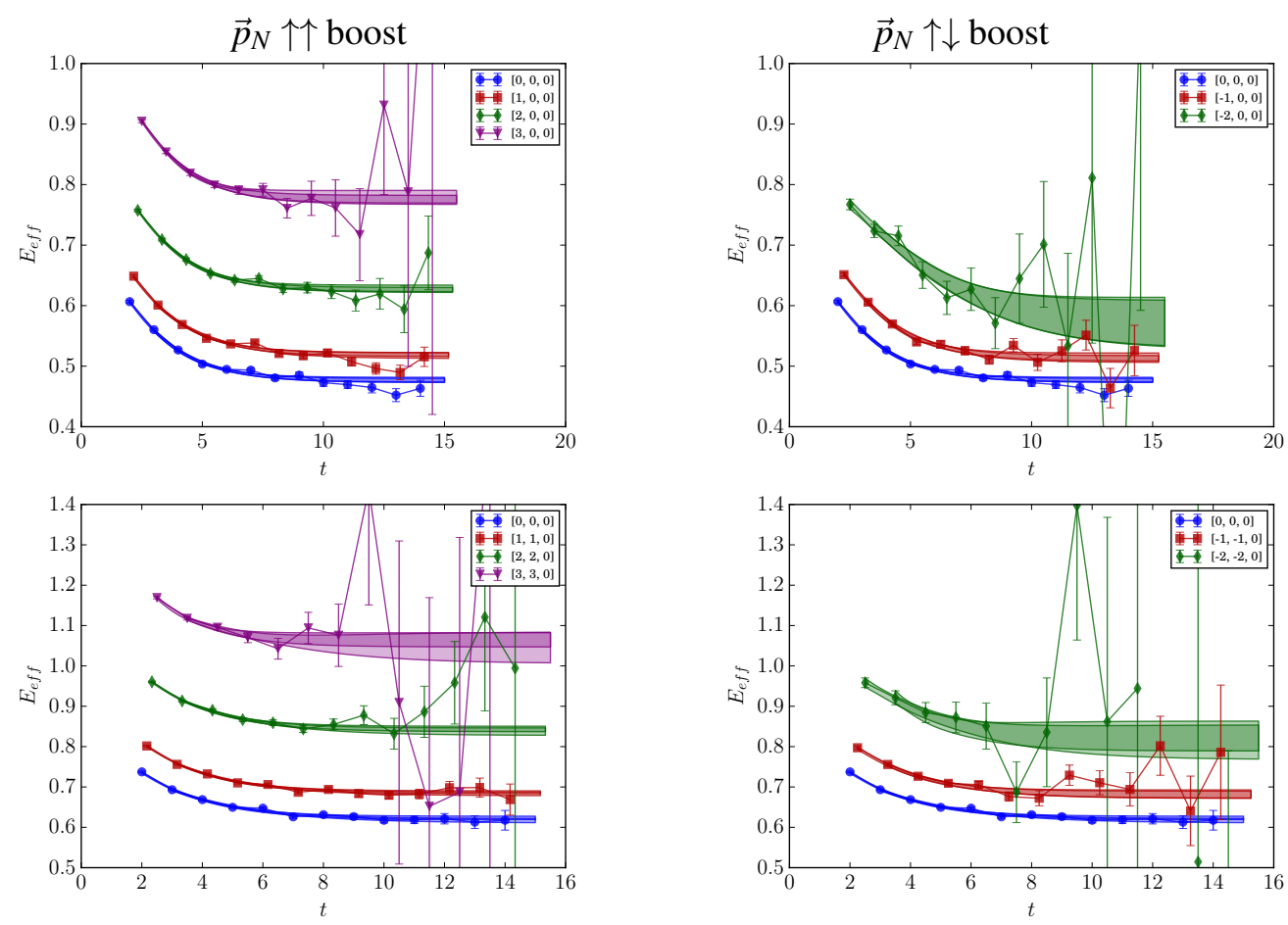

Figure 3: Effective energy plots for nucleon correlators with boosted quark smearing on $a=0.094 \mathrm{fm}$ (top) and $a=0.127 \mathrm{fm}$ (bottom) ensembles. Results are shown for the nucleon momenta $\vec{p}_{N}$ aligned (left) and anti-aligned (right) with the smearing momentum.

source-sink separation. The two-state fits and the "summation" analysis are apparently inadequate to remove these excited states. It is surprising though that while our form factors are apparently affected by excited state contamination, their ratio shows little $t_{\text {sep }}$ dependence shown in Fig.5(left). The relative scaling of the $F_{1}$ and $F_{2}$ form factors shown in Fig. 5(left) agrees qualitatively with the phenomenological prediction [2] $Q^{2} F_{1}\left(Q^{2}\right) / F_{2}\left(Q^{2}\right) \sim \log \left[Q^{2} / \Lambda^{2}\right]$.
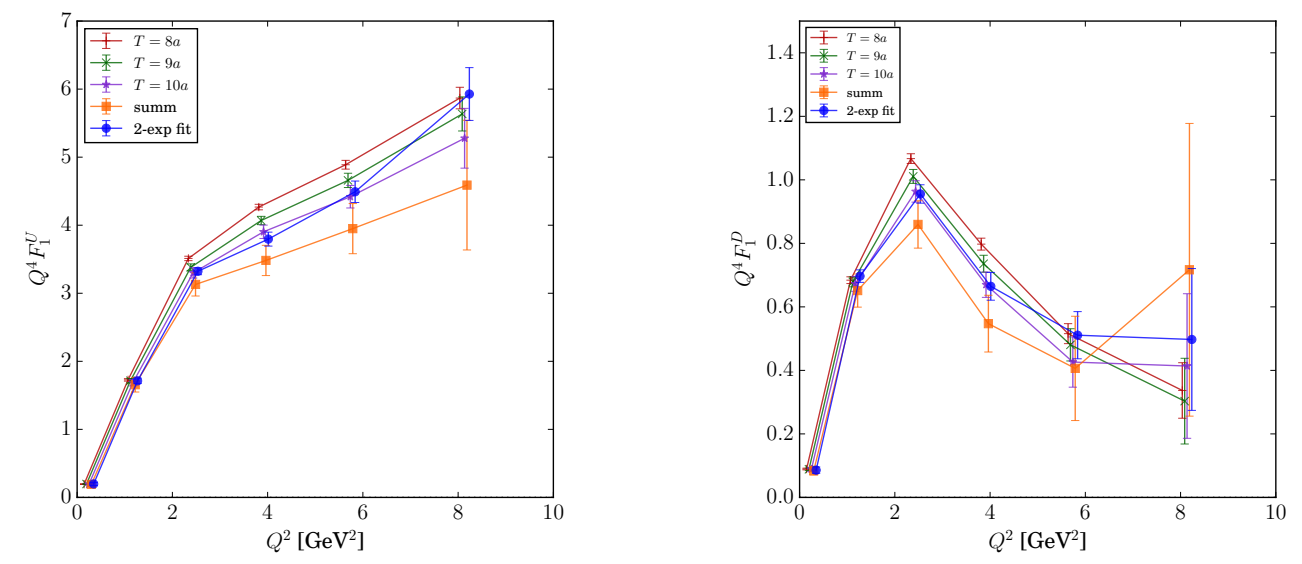

Figure 4: Scaling of form factor $F_{1}$ (connected part) with $Q^{2}$. Scaling of $Q^{2} F_{1}$ for the $u$ (left) and $d$ (right) quarks Data from the $a=0.094 \mathrm{fm}$ ensemble. 

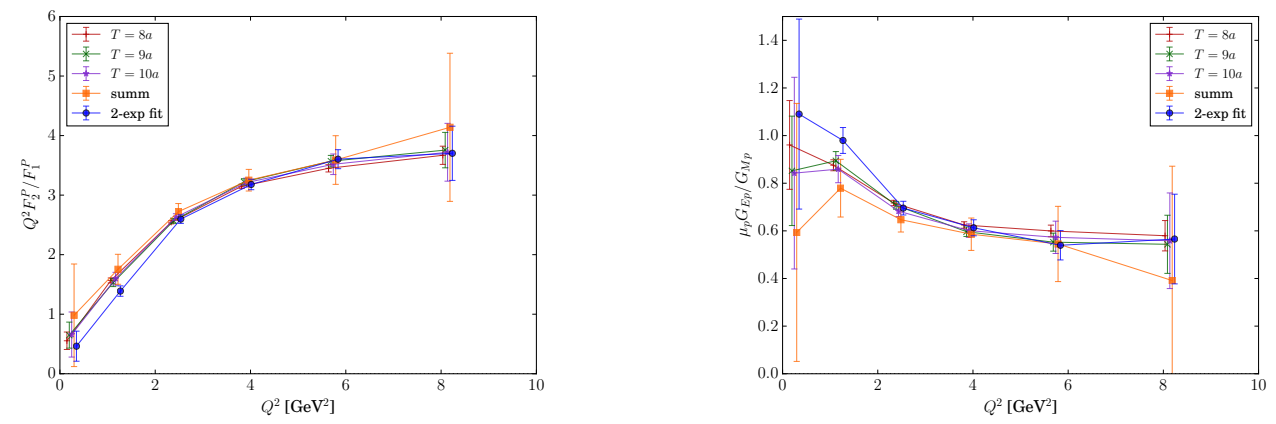

Figure 5: Ratios of form factors (connected parts) for the proton on the $a=0.094 \mathrm{fm}$ ensemble. (Left) the ratio of $Q^{2} F_{2 p} / F_{1 p}$ vs the momentum transfer $Q^{2}$. (Right) the ratio of Sachs form factors $\mu_{p} G_{E p} / G_{M p}$.

In Figure 5(right) we show the ratio of the proton form factors $\mu_{p} G_{E p} / G_{M p}$. From experiments, this ratio is expected to become zero around $Q^{2} \approx 7 \ldots 8 \mathrm{GeV}^{2}$. However, our data does not reproduce this trend. We note however that the zero value of $G_{E p}\left(Q^{2}\right)$ must result from delicate cancellation between the $F_{1 p}$ and $F_{2 p}$ form factors for the proton in $G_{E}=F_{1}-\frac{Q^{2}}{4 M_{N}^{2}}$. Quarkdisconnected contributions to the proton form factors may be crucial to reproduce this cancellation. We show the ratio of disconnected contributions to form factors $F_{1,2}^{\text {disc }}$ to the connected contribution from the $u$-quark $F_{1,2}^{u}$ in Fig. 6 These disconnected contributions have been calculated using hierarchical probing with deflation [9], and the statistics is not yet sufficient to yield statistically significant non-zero values. In the case of $F_{1 p}$, the disconnected contribution is $\lesssim 10 \%$ of connected for $Q^{2} \leq 6 \mathrm{GeV}^{2}$, but its uncertainty is significantly larger at the largest momentum transfer $Q^{2}$ point. In the case of $F_{2 p}$, the disconnected contribution is larger relative to the connected contribution, and may be a substantial correction for the two largest $Q^{2}$ points. We conclude that the ratio $G_{E p} / G_{M p}$ ratio may be affected by the disconnected contributions to the $F_{2 p}$ form factors, in addition to other systematic effects.
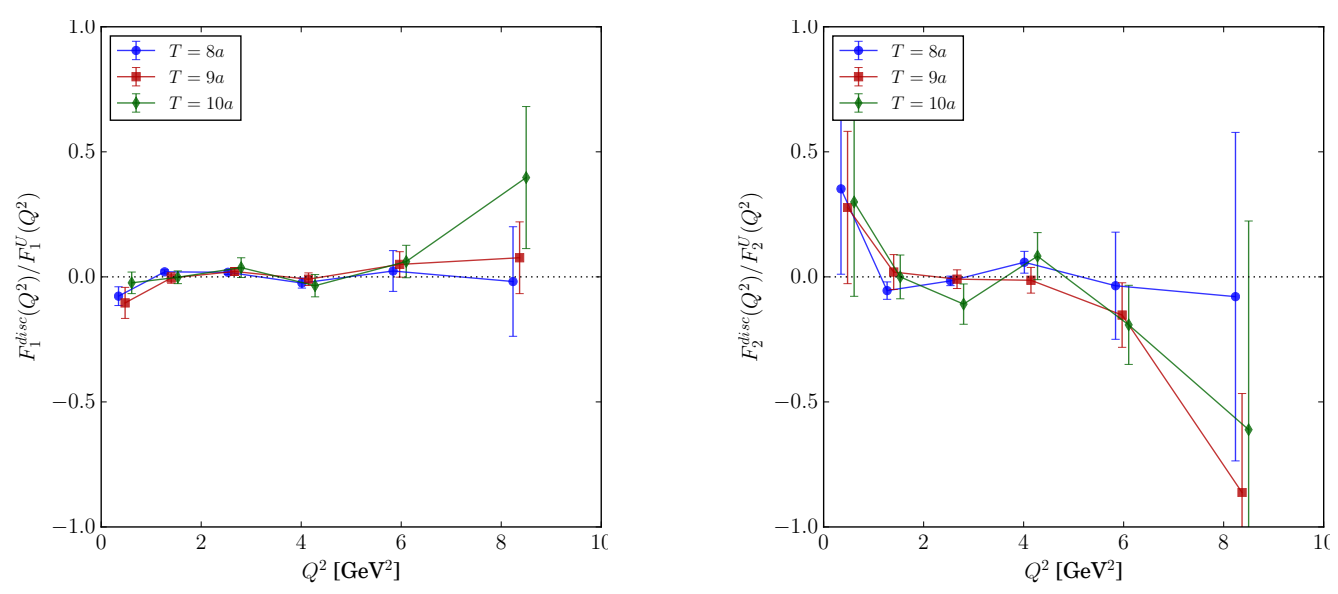

Figure 6: Ratios of disconnected and connected contributions to the vector Dirac $F_{1}$ (left) and Pauli $F_{2}$ (right) form factors vs the momentum transfer $Q^{2}$ on the $a=0.094 \mathrm{fm}$ ensemble. 


\section{Discussion}

We have presented preliminary results for the nucleon form factors at high momentum transfer up to $Q^{2} \approx 6 \mathrm{GeV}^{2}$ calculated using the novel technique of "boosted" quark source smearing.Both connected and disconnected contributions have been calculated, but the statistics are not sufficient to observe any non-zero disconnected contribution to the form factors. Our results suggest that there are significant contributions from excited states, and simple multi-state fits may be inadequate to control them. An interesting observation that the Dirac and Pauli relative form factor scaling $Q^{2} F_{1} / F_{2}$ nevertheless agrees qualitatively with perturbative QCD predictions. This, however, may be explained as a common feature for the ground and the excited states of the nucleon, since perturbative QCD cannot discriminate between them.

The boosted smearing is a substantial help in improving the signal in these calculations. It is likely that the signal may be substantially improved by choosing larger smearing momentum and tuning it following Ref. [6] to maximize the advantage of the technique. In addition, the basis of nucleon interpolating fields has to be extended to include the negative parity states that become important for relativistic nucleon fields [10]. We plan to study the quark current improvement $V_{I}^{\mu}=\bar{q} \gamma^{\mu} q+c_{V} \partial_{v} T^{\mu v}$, which may be especially critical at high momenta because the improvement term contains a derivative.

\section{Acknowledgements}

We thank the JLab group for generating the gauge configurations with dynamical cloverimproved Wilson fermions. Calculations have been performed on USQCD resources at Fermilab. SNS has been supported by the Nathan Isgur fellowship program at JLab and RIKEN BNL Research Center under its joint tenure track fellowship with Stony Brook University. KO and AG have been supported by the U.S. Department of Energy through Grant Number DE-FG02-04ER41302.

\section{References}

[1] S. J. Brodsky and G. R. Farrar, Phys. Rev. D 11, 1309 (1975).

[2] A. V. Belitsky, X.-d. Ji, and F. Yuan, Phys. Rev. Lett. 91, 092003 (2003).

[3] G. D. Cates, C. W. de Jager, S. Riordan, and B. Wojtsekhowski, Phys. Rev. Lett. 106, 252003 (2011).

[4] G. P. Lepage, Invited lectures given at TASI'89 Summer School, Boulder, CO, Jun 4-30, 1989.

[5] B. Musch, 2013, private communications.

[6] G. S. Bali, B. Lang, B. U. Musch, and A. Schäfer, Phys. Rev. D93, 094515 (2016).

[7] B. Yoon et al., Phys. Rev. D93, 114506 (2016).

[8] B. Yoon et al., (2016).

[9] A. S. Gambhir et al., PoS LATTICE2016, 265 (2016).

[10] F. M. Stokes, W. Kamleh, D. B. Leinweber, and B. J. Owen, PoS LATTICE2016, 161 (2017). 